\section{Test, serieller diagnostischer}

\section{R.-D. Hilgers ${ }^{1}$, N. Heussen ${ }^{1}$ und S. Stanzel ${ }^{2}$}

${ }^{1}$ Institut für Medizinische Statistik, Universitätsklinikum der RWTH Aachen, Aachen, Deutschland

${ }^{2}$ DKFZ Heidelberg, Heidelberg, Deutschland

\section{Englischer Begriff serial diagnostic testing}

Definition Unter einem seriellen diagnostischen Test versteht man die nacheinander geschaltete Durchführung mehrerer diagnostischer Tests ( $\triangleright$ Test, diagnostischer), d. h., der Folgetest wird erst durchgeführt, wenn die Resultate der vorangegangenen Tests feststehen.

Beschreibung Im Rahmen der Durchführung multipler diagnostischer Tests ( $\triangleright$ Test, multipler diagnostischer) ist grundsätzlich anzumerken, dass der Entscheidungsregel eine besondere Bedeutung zukommt. Ein vielfach praktiziertes Verfahren beruht auf dem „Believe the positive“-Prinzip. Dabei müssen alle in streng konsekutiver Abfolge durchgeführten Einzeltests ein positives Resultat für die zu ermittelnde Diagnose liefern, um die Diagnose zu bestätigen. Hingegen wird der diagnostische Prozess bei Auftreten eines negativen Ergebnisses beendet. Die serielle Durchführung wird meist angewandt, wenn eine schnelle Beurteilung nicht primär erforderlich ist oder die Tests zu teuer oder risikoreich sind. Gegenüber den Einzeltests erhöht die serielle Durchführung im Allgemeinen die (Gesamt-)Spezifität ( $\triangleright$ Spezifität, diagnostische) und damit den positiven Vorhersagewert ( $\vee$ Vorhersagewert, positiver), verringert jedoch die (Gesamt-)Sensitivität ( $\vee$ Sensitivität, diagnostische) und den negativen Vorhersagewert ( $\triangleright$ Vorhersagewert, negativer). Daraus resultiert insgesamt ein erhöhtes Risiko, die Krankheit zu übersehen, bei gleichzeitig erhöhter Sicherheit für eine Bestätigung der Krankheit durch ein positives Testresultat.

Das $\triangleright$ a posteriori odds eines seriellen diagnostischen Tests lässt sich aus dem $\triangleright$ a priori odds bei Kenntnis der Sensitivitäten und Spezifitäten der Einzeltests ermitteln durch Multiplikation der einzelnen positiven Likelihood Ratios ( $\triangleright$ Likelihood Ratio, positives).

\section{Literatur}

Hilgers R-D, Bauer P, Scheiber V (2002) Einführung in die Medizinische Statistik. Springer, Berlin/Heidelberg/New York 